\title{
DEMONSTRAÇÃO DE UM SOFTWARE QUE UTILIZA O ALGORITMO NAIVE BAYES PARA IDENTIFICAÇÃO DE RADIONUCLÍDEOS ATRAVÉS DE ESPETROSCOPIA GAMA.
}

\author{
Márcio Magalhães de Andrade Silva \\ Instituto Militar de Engenharia - IME \\ Praça Gen. Tibúrcio, 80 - Urca, Rio de Janeiro - RJ \\ marcio.magalhaes.br@gmail.com \\ Gabriela Martins Duarte \\ Instituto Militar de Engenharia - IME \\ Praça Gen. Tibúrcio, 80 - Urca, Rio de Janeiro - RJ \\ gabrimduarte92@gmail.com \\ Domingos Oliveira Cardoso \\ Instituto Militar de Engenharia - IME \\ Praça Gen. Tibúrcio, 80 - Urca, Rio de Janeiro - RJ \\ domingos.oliveiralvr71@gmail.com \\ Clóves Júnior Fonseca \\ Instituto Militar de Engenharia - IME \\ Praça Gen. Tibúrcio, 80 - Urca, Rio de Janeiro - RJ \\ cjfonseca16@gmail.com \\ Caio Caraciolo Rodrigues Elias \\ Instituto Militar de Engenharia - IME \\ Praça Gen. Tibúrcio, 80 - Urca, Rio de Janeiro - RJ \\ caioelias@poli.ufrj.br
}

\section{RESUMO}

No mundo atual presencia-se a confluência de tecnologias e áreas do conhecimento. O grau de automação nas diversas aplicações industriais além do uso da inteligência artificial se fazem presentes, cada vez mais, nas linhas de produção. Dentro da indústria nuclear, procuram-se oportunidades de aplicação dos mecanismos de segurança, a fim de automatizar o monitoramento dos materiais radioativos como, por exemplo, os provenientes de rejeitos de processos ligados ao ciclo do combustível nuclear. Nesse contexto, é de grande serventia o desenvolvimento de um software capaz de alarmar quando detectada uma variação na assinatura espectroscópica. Quando este determinar alguma diversidade, sendo capaz de auxiliar na tomada de decisão em situações de emergência radiológica. Diante disso, o presente estudo levanta a viabilidade da elaboração de um software (desenvolvido em JAVA), capaz de identificar, automaticamente, um isótopo através do levantamento de seu espectro gama.

Palavra-chave: Espectroscopia; Inteligência Artificial; Naive Bayes; WEKA 3; Identificação de Radionuclídeos. 


\begin{abstract}
In the present world there is a confluence of technologies and areas of knowledge. The degree of automation in various industrial applications as well as the use of artificial intelligence is increasingly present in industrial production lines. Inside the nuclear industry, opportunities are sought for safety mechanisms to be applied in order to automate the monitoring of radioactive materials such as those from nuclear fuel cycle process tailings. In this context, it is very useful to develop software capable of alarming when a variation in the spectroscopic signature is detected. When this determines some diversity, being able to assist in decision making in radiological emergency situations. Given this, the present study raises the feasibility of developing a software (developed in JAVA), capable of automatically identifying an isotope by surveying its gamma spectrum.
\end{abstract}

Keywords: Spectroscopy; Artificial intelligence; Naive Bayes; WEKA 3; Radionuclide Identification.

\title{
Como Citar:
}

SILVA, M. M. A. et al. Demonstração de um Software que Utiliza o Algoritmo Naive Bayes para Identificação de Radionuclídeos Através de Espetroscopia Gama. In: SIMPÓSIO DE PESQUISA OPERACIONAL E LOGÍSTICA DA MARINHA, 19., 2019, Rio de Janeiro, RJ. Anais 2019. Rio de Janeiro: Centro de Análises de Sistemas Navais, 2019.

\section{INTRODUÇÃO}

No mundo atual presencia-se a confluência de tecnologias e áreas do conhecimento. O grau de automação nas diversas aplicações industriais e o uso da inteligência artificial se fazem presentes, cada vez mais, nas linhas de produção.

Dentro da indústria nuclear, procuram-se oportunidades de aplicação dos mecanismos de segurança, a fim de automatizar o monitoramento dos materiais radioativos como, por exemplo, os provenientes de rejeitos de processos ligados ao ciclo do combustível nuclear.

Nesse contexto, o desenvolvimento de um software capaz de alarmar quando detectada uma variação no espectro gama, para um determinado inventário, é de grande valia no auxílio à tomada de decisão, em especial em situações de emergência radiológica.

Diante disso, o presente estudo apresenta a viabilidade do desenvolvimento de um software, baseado em aprendizagem supervisionada de máquina, capaz de identificar, automaticamente, um isótopo através do levantamento de seu espectro gama. Demonstra-se um protótipo de aplicativo classificador, que utiliza o algoritmo Naive Bayes para a identificação de um dentre três espectros conhecidos. O software é desenvolvido em JAVA, com a utilização da biblioteca de inteligência artificial WEKA 3. 


\section{REVISÃO DA LITERATURA}

Os algoritmos baseados em aprendizagem supervisionada de máquina têm aplicações diversas tanto na avaliação operacional quanto no auxílio à tomada de decisão em inúmeras áreas do conhecimento. Áreas como medicina, educação, agricultura, mercado financeiro, entre outras, são alvos de estudos que focam a melhoria de processos, conforme mostrado a seguir.

Um exemplo de aplicação voltado à cafeicultura é o uso de algoritmos de classificação no auxílio à análise de imagens provenientes de sensoriamento remoto, com a finalidade de mapeamento e monitoração das lavouras de café, conforme o trabalho de Souza, C.G. et al (2016).

Há, também, exemplos de aplicação de aprendizagem de máquina em modelos preditivos para detecção de diabetes, e a análise comparativa entre o desempenho de vários algoritmos de inteligência artificial dentro desse contexto, segundo OLIVERA, A. R. et al (2017).

Ainda na área médica, SILVA, F. R. et al. (2017) demonstrou o uso de aprendizagem de máquina em um estudo observacional que visou avaliar a sensibilidade e a especificidade desse tipo de algoritmo aplicado ao diagnóstico de glaucoma, com o uso de Spectral Domain OCT (SD-OCT) e perimetria automatizada acromática (PAA).

A aplicação de inteligência artificial na engenharia nuclear originou algumas teses de doutorado, como no trabalho de SILVEIRA, P. C. R. (2017) que descreve o desenvolvimento de um sistema robótico autônomo. Este sistema utiliza a metodologia de aprendizagem ativa de processos gaussianos combinados com computação evolucionária.

Outra aplicação de inteligência artificial na engenharia nuclear está descrita na tese de Oliveira, I. M. S. (2013), onde se demonstrou o uso do Algoritmo de Otimização por Colônia de Abelhas Artificiais (Artificial Bee Colony) para otimizar o problema de recargas de reatores PWR (Pressurized Water Reactor).

\section{METODOLOGIA}

A partir do espectro inicial das fontes disponíveis no Laboratório Nuclear do Instituto Militar de Engenharia (IME), em especial Cobalto-60 (60Co), Césio-137 (137Cs) e Európio-152 (152Eu), utilizou-se dois detectores: um de Germânio Hiperpuro (HPGe) e um do tipo cintilador (NaI), para levantar os espectros junto com o software Maestro. Por meio dos espectros iniciais, a aplicação desenvolvida seguiu dois macroprocessos: (i) a construção da base de conhecimento e (ii) a classificação de novos espectros.

A construção da base de conhecimento objetiva o estabelecimento de uma base de dados histórica para que o software embase suas decisões. Já a classificação, consiste na submissão de um novo espectro, que não faz parte da base de conhecimento.

Antes de ser armazenado, o espectro capturado com 8912 canais é amostrado para 256 canais, visando à diminuição do custo computacional no processamento dos dados. A segunda operação realizada com o espectro, quando este já se encontra carregado no banco de dados, é a normalização dos valores de contagem de cada um deles, para que todos os espectros processados estejam na mesma escala, visando novamente a redução do custo computacional. O último passo antes da geração do arquivo ARFF, usado como base de conhecimento do algoritmo de classificação, é a discretização dos canais dos vetores de dados simulados. O algoritmo Naive Bayes utiliza atributos nominais e não numéricos para classificação, sendo assim, é necessária a manipulação dos dados dos espectros simulados no sentido de discretizá-los.

Uma vez realizado o processo de discretização, a aplicação gera o arquivo ARFF, passando a um novo estado, no qual estará apta à classificação de arquivos de entrada. Estes arquivos passam pelos mesmos processos dos espectros presentes na base de conhecimento, a fim de facilitar a comparação. 


\section{RESULTADOS}

O primeiro teste realizado com o software, foi feito utilizando como base de conhecimento os dados normalizados e discretizados obtidos experimentalmente para os nuclídeos ${ }^{60} \mathrm{Co},{ }^{137} \mathrm{Cs} \mathrm{e}$ ${ }^{152} \mathrm{Eu}$, e obteve como resultado final a composição do arquivo ARFF.

Para validar a acurácia do classificador baseado no algoritmo Naive Bayes, foram submetidos os espectros dos três nuclídeos, obtidos do arquivo exportado diretamente do software Maestro, sem qualquer tipo de tratamento. Os arquivos SPE utilizados na validação são os mesmos usados para compor a base de treinamento do algoritmo. Além do espectro, a cada entrada foi fornecida a informação do tipo de detector envolvido (HPGe ou NaI).

Conforme esperado, o software obteve sucesso na classificação dos espectros utilizados no treinamento do algoritmo. A Figura 1 mostra a tela de entrada do arquivo SPE e a Figura 2 o resultado da classificação, dado na forma da probabilidade do arquivo importado se referir a um dos três elementos. Tanto nos espectros obtidos através do cintilador NaI quanto nos obtidos pelo HPGe, os três resultados apresentados pelo software, estimam em $100 \%$ a probabilidade da amostra ser do nuclídeo usado na validação. No caso do HPGe foram testados ainda os mesmos espectros com a subtração do espectro da radiação de background, e, ainda sim, os resultados foram idênticos.

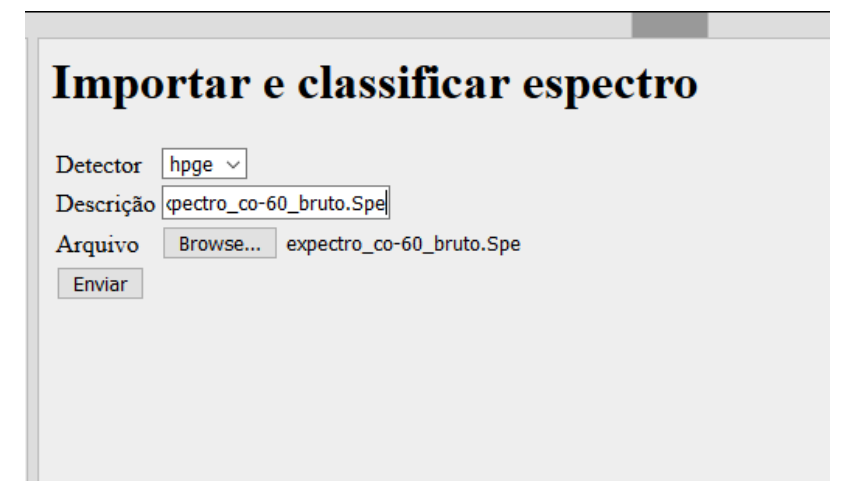

Figura 1 - Tela de importação do arquivo SPE.

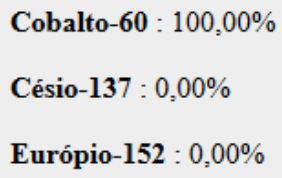

Figura 2 - Resultado da classificação.

Após a validação, com o mesmo arquivo ARFF usado como treinamento, espectros obtidos em condições experimentais diferentes, foram submetidos à classificação do software. Ao submeter o Césio-137, o software classificou erroneamente a amostra como tendo a maior probabilidade de ser Európio-152, de acordo com as probabilidades exibidas na Figura 3. Contudo, o erro do classificador, neste caso, indica capacidade do software em detectar as diferenças na assinatura espectrométrica de uma instalação, contribuindo para a proteção radiológica da mesma.

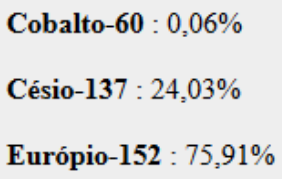

Figura 3 - Classificação incorreta do Césio-137. 
O Európio-152, no segundo experimento, foi classificado corretamente, assim como o Cobalto-60. Ambos foram classificados como tendo 100\% de probabilidade de serem os isótopos corretos.

Em um terceiro experimento, os arquivos SPE que continham os espectros submetidos à classificação no experimento anterior, foram incorporados ao arquivo ARFF para o treinamento do classificador. Logo após, estes espectros foram submetidos novamente à classificação. $\mathrm{O}$ espectro de Césio-137, classificado de forma incorreta anteriormente, dessa vez foi classificado corretamente, conforme ilustrado na figura 4.

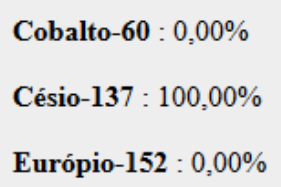

Figura 4 - Resultado após ampliação da base de conhecimento.

Os espectros de Césio-137 e Cobalto-60 utilizados no experimento anterior, assim como os seis espectros utilizados na primeira experiência (obtidos nos detectores do tipo HPGe e NaI para cada um dos três nuclídeos estudados) de validação do software também tiveram sua classificação realizada de forma adequada.

\section{CONCLUSÃO}

Procurou-se demonstrar que a confluência de tecnologias, como a espectroscopia gama e a inteligência artificial (baseada em aprendizagem supervisionada de máquina), podem ser integradas com o uso de um software desenvolvido em JAVA, utilizando um classificador bayesiano contido na biblioteca WEKA 3. Verificou-se ainda que, através do fornecimento de uma base de conhecimento, este software é capaz de reconhecer o espectro padrão gama de cada um dos radionuclídeos utilizados para teste. Desse modo, as inspeções podem ser realizadas de duas maneiras: (i) automática, quando o software é programado e executa as verificações online; (ii) inspeções periódicas, quando em um intervalo pré-determinado as verificações são executadas, por uma equipe treinada para tal fim.

Os experimentos realizados demonstram o funcionamento do algoritmo classificador bayesiano, denominado Naive Bayes, na análise e classificação de dados previamente amostrados, normalizados e discretizados, conforme demonstrado na seção 3. Demonstrou-se a versatilidade do método, uma vez que ao falhar na classificação do espectro de Césio-137, bastou ampliar a base de treinamento do ARFF, para o algoritmo Naive Bayes "aprender" e classificar corretamente a nova amostra.

Dessa forma, embora em alguns casos o clarificador bayesiano se torne errático, espera-se que quando fornecida uma extensa base de treinamento, mantendo condições de boa geometria e blindagem adequada da radiação de fundo, é possível obter resultados que atendam o objetivo - da automação de monitoramento ambiental, checagem dos limites existentes no inventário, além da caracterização da identidade espectrométrica - proposto nesse artigo. 


\section{REFERÊNCIAS BIBLIOGRÁFICAS}

DE OLIVEIRA, I. M. Inteligência de enxames aplicada ao problema de otimização de recargas de reatores nucleares a água pressurizada. Universidade Federal do Rio de Janeiro. 2013. Disponível em:

$<$ http://antigo.nuclear.ufrj.br/DScTeses/teses2013/Tese_Iona_Maghali.pdf $>$ Acesso em: 29 mai. 2019.

KNOLL, G. F. Radiation Detection and Measurements. New York: John Wiley \& Sons Inc, 1989. 3 ed. 816 p. ISBN 0-417-07338-5.

OLIVERA, A. R. et al. Comparison of machine-learning algorithms to build a predictive model for detecting undiagnosed diabetes-ELSA-Brasil: accuracy study. Sao Paulo Medical Journal, v.135, n.3, p. 234-246, 2017. Disponível em:

$<$ http://www.scielo.br/scielo.php?script $=$ sci_arttext\&pid $=$ S151631802017000300234\&lng=en\&nrm=iso $>$. Acesso em: 29 mai. 2019.

SILVA, F. R. et al. Sensitivity and specificity of machine learning classifiers for glaucoma diagnosis using Spectral Domain OCT and standard automated perimetry. Arquivos brasileiros de oftalmologia, v. 76, n. 3, p. 170-174, 2013. Disponível em: $<$ http://www.scielo.br/scielo.php?script=sci_arttext\&pid=S000427492013000300008\&lng=en\&nrm=iso $>$. Acesso em: 29 mai. 2019.

SILVEIRA, P. C. R. Sistema robótico autônomo para mapeamento de taxas de dose utilizando aprendizado ativo de processos gaussianos e computação evolucionária. Universidade Federal do Rio de Janeiro. 2017. Tese de Doutorado. Disponível em: $<$ http://www.con.ufrj.br/wp-content/uploads/2018/02/Tese-Paulo-Cezar-RochaSilveira.pdf> Acesso em: 29 mai. 2019.

SOUZA, C. G. et al. Algoritmos de aprendizagem de máquina e variáveis de sensoriamento remoto para o mapeamento da cafeicultura. Boletim de Ciências Geodésicas, v. 22, n. 4, p.751-773. 2016. Disponível em: <http://www.scielo.br/scielo.php? script=sci_arttext\&pid=S1982-21702016000400751\&lng=en\&nrm=iso $>$ Acesso em: 29 mai. 2019.

TSOULFANIDIS, N., LANDSBERGER, S. Measurement \& Detection of Radiation. Boca Raton: Taylor \& Francis Group, 2015. 4 ed. 595 p. ISBN-13:978-1-4822-1548-9.

WEKA 3: Machine learning software in JAVA. The University of Waikato (NZ). Disponível em: $<$ https://www.cs.waikato.ac.nz/ml/weka/> Acesso em: 29 mai. 2019. 